\title{
Real-Time Mobile Personalized Simulations of Impaired Colour Vision
}

\author{
Rhouri McAlpine \\ University of Dundee \\ Dundee, Scotland, UK \\ r.mcalpine@dundee.ac.uk
}

\author{
David R. Flatla \\ University of Dundee \\ Dundee, Scotland, UK \\ d.flatla@dundee.ac.uk
}

\begin{abstract}
Colour forms an essential element of day-to-day life for most people, but at least $5 \%$ of the world have Impaired Colour Vision (ICV) - seeing fewer colours than everyone else. Those with typical colour vision find it difficult to understand how people with ICV perceive colour, leading to misunderstanding and challenges for people with ICV. To help improve understanding, personalized simulations of ICV have been developed, but are computationally demanding (so limited to static images), which limits the value of these simulations. To address this, we extended personalized ICV simulations to work in real time on a mobile device to allow people with typical colour vision greater freedom in exploring ICV. To validate our approach, we compared our real-time simulation technique to an existing adjustable simulation technique and found general agreement between the two. We then deployed three real-time personalized ICV simulations to nine people with typical colour vision, encouraging them to take photos of interesting colour situations. In just over one week, participants recorded over 450 real-world images of situations where their simulation presented a distinct challenge for their respective ICV participant. Through a questionnaire and discussion of photos with participants, we found that our solution provides a valuable mechanism for building understanding of ICV for people with typical colour vision.
\end{abstract}

\section{Keywords}

Impaired colour vision; colour vision deficiency; colourblindness; mobile personalized simulation

\section{INTRODUCTION}

In a recent topical thread on Reddit discussing poor treatment by employers ${ }^{1}$ a contributor relayed this experience:

I'm colorblind. My boss assigned me a work task once that was color-coded in a way that I

1 https://redd.it/4fzph7

Permission to make digital or hard copies of part or all of this work for personal or classroom use is granted without fee provided that copies are not made or distributed for profit or commercial advantage and that copies bear this notice and the full citation on the first page. Copyrights for third-party components of this work must be honored. For all other uses, contact the owner/author(s).

ASSETS '16 October 23-26, 2016, Reno, NV, USA

(C) 2016 Copyright held by the owner/author(s).

ACM ISBN 978-1-4503-4124-0/16/10.

DOI: http://dx.doi.org/10.1145/2982142.2982170 couldn't see. When I brought up my vision issues - not refusing to do the work, but asking for accommodation - I was written up for what they perceived as "insubordination".

When I filed a complaint with HR asking for the write-up to be removed, the HR rep "graciously" gave me what she perceived as a solution: that I should go use the company's Tuition Reimbursement program to go take a remedial art class at my local community college. "So that you can finally learn your colors", she said.

Yes, it's Reddit, but the Internet abounds with similar stories of others' lack of awareness of what it is like living with Impaired Colour Vision (ICV - commonly called colourblindness) ${ }^{2}$ Similar challenges have been documented in the academic literature $5,27,26,15$.

Accounts documenting the day-to-day challenges faced by people with ICV often reveal a lack of awareness among people with typical colour vision about what people with ICV actually perceive. Indeed, there seems to be a range of severities of this lack of awareness, from those who are entirely unaware that ICV exists, to those who have a general understanding of the colours that are indistinguishable for someone with ICV, but little comprehension of how the visual experience of people with ICV differs from their own.

A recent survey of 27 web designers found that $25 / 27$ (93\%) of respondents were aware of the existence of ICV, suggesting that the number of people who are entirely unaware of ICV is low. However, the same study found that substantially fewer $(13 / 27-48 \%)$ respondents considered end users with ICV when working on a project, with $17 / 27$ $(63 \%)$ of respondents considering end users with ICV 'never' or 'rarely' 29]. Although many factors likely influence a designer's decision to consider end-user abilities (e.g., contract constraints), it is possible that these designers also lack understanding of how ICV influences colour perception, leading to a lack of accommodation in their designs.

In order to help improve awareness of how people with ICV perceive colours, simulations of ICV have been proposed $20,17,30,4,19$ in which an image's colours are modified to illustrate how those same colours appear for someone with ICV. These simulation techniques provide an approximate representation of experiencing ICV, but lack the ability to precisely represent an individual's unique colour per-

\footnotetext{
2 www.colourblindawareness.org gives one work-related account and provides others. https://redd.it/310n73 is a more general conversation about ICV challenges. Www.colorblindness.com lists many challenges related to ICV.
} 
ception abilities due to a lack of precise diagnosis of ICV or the presence of simultaneous or compound ICVs. To address these limitations, personalized simulations of ICV have been developed 11, but are computationally demanding and have only been applied to preselected images in a lab environment. As a result, personalized simulations are not available to our original Reddit contributor's co-workers (boss and HR rep) to help them understand exactly how their colleague sees the world.

To address this, we extended personalized simulations of ICV to operate in real time on mobile platforms. To do this, we build an Android app that: 1) measures an ICV individual's colour perception abilities to produce a personalized simulation, and 2) applies the personalized simulation to the live video feed from the device's camera and displays the ICV-simulated video feed on the device's screen. The result is a 'magic window' application that allows someone with typical colour vision to see real-world colours as someone with ICV by peering through the 'magic window'.

To accomplish this, the colour perception abilities of someone with ICV are captured via a calibration task 9]. The resulting values are used to execute the personalized simulation algorithm (described in [11]) on an image containing every RGB666 colour to generate a Look-Up Table (LUT) that maps original RGB values to personalized simulation RGB values. We use the LUT in OpenCV and RenderScript to modify each pixel of each frame from the video camera in parallel, then draw the simulated frame to the screen.

In addition to providing personalized simulations, we also corrected an error in the original algorithm (as identified in [11]), and added standard (i.e., non-personalized) simulations for common severe types of ICV to our application.

We evaluated our simulation application in two ways. First, we compared our corrected simulation algorithm to an existing adjustable ICV simulation technique (described in [19]) to understand if our personalized simulation technique agreed with other simulation techniques. We found some differences between the approaches, but overall our simulation technique generally agreed with the adjustable technique.

Second, we generated personalized ICV simulations for three people with ICV (one protan, one deutan, one nearmonochromatic), and deployed our app with these simulations to nine people with typical colour vision. We added a 'capture image' feature to our simulation app that allowed participants to capture both the original and simulated current image at any given time. Participants had the simulation for 7-10 days, and gathered 461 images representing a diverse set of situations. Participants with typical colour vision reported increased understanding of the visual abilities of their particular ICV participant.

This paper makes three contributions. First we correct existing personalized ICV simulations, and describe the first ever real-time mobile personalized simulation of ICV. Second, we compare our personalized ICV simulation technique to an existing adjustable ICV simulation technique, identifying future extensions of our approach. Third, we present empirical data suggesting that real-time, mobile personalized simulations of ICV help improve ICV awareness for people with typical colour vision. In combination, our contributions provide a new opportunity to help improve general awareness of ICV.

\section{BACKGROUND \& RELATED WORK}

\subsection{Impaired Colour Vision}

Commonly referred to as colourblindness or Colour Vision Deficiency (CVD), Impaired Colour Vision (ICV) affects anyone who has difficulty discriminating between colours that the majority of people can differentiate 8. The terms 'colourblindness' and 'CVD' typically apply to only the inherited forms of ICV; but the term 'ICV' includes three broad classes of this impairment: inherited, acquired, and situationally induced [10].

Three types of retinal cone cells are responsible for typical human colour vision; long $(l)$, medium $(m)$, and short $(s)$ wavelength sensitive cones respond to varying ranges of electromagnetic radiation between 700nm and 380nm 31. Colour is perceived when light differentially stimulates the cone cells. Differential stimulation of the $l$ and $m$ cones allows discrimination between reds and greens; differential stimulation of a combined $l+m$ and the $s$ cones allows discrimination between yellows and blues 28.

Inherited ICV affects the $l$ and $m$ cones in over $99 \%$ of cases, resulting in reduced discriminability between colours that differ in their respective amounts of red and/or green (e.g., green and orange, blue and purple) 2]. The taxonomy of inherited ICVs include dichromacy (one cone type absent; $1 / 4$ of cases) - protanopia (missing $l$ cones), deuteranopia (missing $m$ cones), and tritanopia (missing $s$ cones) and its less-severe but more common form anomalous trichromacy (3/4 of cases) - protanomalous ( $l$-cone sensitivity shifted toward $m$-cone sensitivity), deuteranomalous ( $m$-cone shifted toward $l$-cone), and tritanomalous ( $s$-cone shifted toward $m$-cone). In more severe cases, two cones (cone monochromacy) or three cones (rod monochromacy) may be missing, leading to total absence of colour vision 3, 5]. Inherited ICV affects approximately $8 \%$ of males and $0.5 \%$ of females in Caucasians, with variable rates in other populations 3].

In addition to inheritance, ICV can also be acquired either permanently or temporarily. Permanent causes of acquired ICV are typically related to trauma either to the eye (e.g., diabetic retinopathy [12], glaucoma 21], exposure to solvents 7 ]) or to the brain (e.g., stroke or aneurysm leading to cerebral achromatopsia 23]). Acquired ICV can also be temporary in cases where it is induced by medication (e.g., Viagra 33], antidepressants [18]), or in cases of temporary inflammation in the visual system (e.g., optic neuritis 24]). In most cases of trauma to the eye, the individual's ability to discriminate between blues and yellows is reduced [12, but acquired ICV can lead to a mix of blue-yellow and redgreen impairment 24, and even occassionally total loss of colour vision, as in cerebral achromatopsia 23]. ICV acquired as a person ages is typically less severe than other forms of acquired ICV [16], but is much more common one study found that $64 \%$ of British over- 65 s and $32 \%$ of African over-40s 6] had some degree of acquired ICV.

Situationally-induced ICV occurs when any environmental factor inhibits a person's colour differentiation abilities. When using a screen, this can occur when environmental lighting is high or screen brightness is low 22 .

\subsection{Existing ICV Simulation Apps}

Searching on the Google Play Store or Apple's iTunes Store for 'colorblind' turns up a number of mobile apps that provide real-time simulations of ICV (e.g., Color Blind Pal 
by Vincent Fiorentini on iOS and Android). However, every app we have found only provides simulations of dichromacy (one missing cone), so can not simulate the full range of types and severities of ICV that exist because they do not provide a personalized simulation.

\subsection{ICV Simulation}

Almost all attempts at simulating ICV have focused on dichromatic or anomalous trichromatic inherited ICV. The earliest simulation work 20, 17, 4]) defined the mechanism still in use today, described now.

The theoretical approach to simulate inherited dichromatic ICV is to represent each colour as a tristimulus value that represents the degree to which that colour stimulates the three types of cones $(l, m, s)$ 20]. Once a colour is translated into this $l-m-s$ colour space, the colour information encoded by the missing cone is removed or replaced with the information from another cone, thereby effectively reducing the dimensionality of the $l-m$-s representation of any colour from three to two dimensions 4].

In practical terms, this manifests as 'shifting' colour within a colour space such that a three-dimensional colour space is compressed into two dimensions. Using the CIE $\mathrm{L}^{*} \mathrm{u}^{*} \mathrm{v}^{*}$ colour space, which has a luminance $\left(L^{*}\right)$ axis running from black to white, a $u^{*}$ axis from greenish to reddish, and a $v^{*}$ axis running from blue to yellow, simulating protan or deutan inherited ICV leads to a compression of the $\mathrm{u}^{*}$ axis (red-green) toward the $\mathrm{v}^{*}$ axis (yellow-blue) [4, 11.

When simulating anomalous trichromacy (or other partial ICVs), the degree of colour space compression is modulated by the severity of anomalous trichromacy - lower severity gives less compression, greater severity leads to more compression. Machado's adjustable ICV simulation technique 19 takes as input the degree of anomalous cone sensitivity shift, and compresses the colour space accordingly. Flatla and Gutwin's personalized ICV simulations [11] measure the colour differentiation abilities of the person whose vision is to be simulated, and uses these measurements to determine the magnitude of a fixed-distance shift within the CIE L* $\mathrm{u}^{*} \mathrm{v}^{*}$ perceptually-uniform colour space. Our simulation technique is based on the personalized technique, so we next describe how we extended it to mobile.

\section{MOBILE PERSONALIZED ICV SIMULA- TIONS}

The personalized ICV simulation technique described in 11 performs the simulation colour shifting described above on a per-pixel (per-colour) basis. As mentioned earlier, this is computationally demanding, thereby preventing the simulation technique from running in real time.

\subsection{Moving to Real Time}

The computational overhead of the original personalized simulation technique is attributable to the algorithm employed. This algorithm uses a two-pass approach in which a 'primary ICV' is simulated first (either red-green or blueyellow) and then a 'secondary ICV' (opposite of the primary ICV) is simulated using the output from the primary simulation. The primary and secondary ICVs are to accommodate people who have multiple or compound ICVs (e.g., inherited protanomalous with age-related ICV).

For given primary and secondary ICVs and their respective severities, plus an input colour (in RGB), the algorithm:
1. Converts the input RGB colour to CIE L* $\mathrm{u}^{*} \mathrm{v}^{*}$.

2. Determines the $\mathrm{L}^{*} \mathrm{u}^{*} \mathrm{v}^{*}$ primary ICV dichromatic simulation colour for the input colour (using a pre-calculated look-up-table - LUT).

3. Creates a sphere around the input colour in $\mathrm{L}^{*} \mathrm{u}^{*} \mathrm{v}^{*}$ space with radius equal to the input severity.

4. Casts a ray from the input colour to its dichromatic version in $\mathrm{L}^{*} \mathrm{u}^{*} \mathrm{v}^{*}$.

5. Solves the parametric equation for the intersection between the ray and the surface of the sphere.

6. Identifies the $\mathrm{L}^{*} \mathrm{u}^{*} \mathrm{v}^{*}$ colour at the ray-sphere intersection point.

7. Repeats Steps 2-6 once more for the secondary ICV, but using the intersection $\mathrm{L}^{*} \mathrm{u}^{*} \mathrm{v}^{*}$ colour as the input.

8. The intersection $\mathrm{L}^{*} \mathrm{u}^{*} \mathrm{v}^{*}$ colour from the second pass is the final personalized ICV simulation colour.

Although no single step in the above algorithm is egregiously demanding in terms of processing power or memory, the combined effect of all of the steps results in a simulation technique that cannot operate in real-time. Processing a single $512 \times 512$ image requires approximately $1.3 \mathrm{~s}$ on a $2.8 \mathrm{GHz}$ i7 mid-2015 MacBook Pro with 16 GB RAM - a relatively small image, but not nearly real-time performance.

Our solution to this is relatively simple - we extended the use of Look-Up-Tables (LUTs) from storing the precalculated dichromatic simulation colours for all possible input RGB colours to also storing the personalized simulation results for all possible input RGB colours. For a given participant's primary and secondary ICVs and respective severities, we calculate their personalized simulation colours for every RGB colour, and store the results in an image file that serves as our LUT. We use the RGB value for a given input pixel as the index into the LUT image's pixel array to access that pixel's pre-computed simulation colour. We are currently exploring shaders to further improve performance.

\subsection{Correcting Previous Algorithm}

As pointed out in the Discussion section of [11], the personalized ICV simulation algorithm presented there contains a bug that manifests when the severity of both the primary and secondary ICVs is high. The original algorithm utilized the standard dichromatic simulation during the second pass (secondary ICV). However, this sometimes results in the colour-space compression from the primary ICV becoming 'undone' by the secondary ICV compression on account of the radial direction of compression inherent in ICV simulations. As a result, the simulation for someone with monochromatic ICV incorrectly contains colours.

To address this, we incorporated an additional shifting mechanism in which the dichromatic version of the input colour for the second stage is shifted according to the primary ICV's type and severity. This results in truly monochromatic ICV simulation when the severities for the primary and secondary ICVs are both high. 


\subsection{Mobile Calibration}

In order to provide personalized ICV simulations, the colour differentiation abilities of people with ICV need to be captured. To do this, we ported the calibration procedure used in the original personalized ICV simulation technique 11. (originally described in 9]) directly to mobile. A screenshot of the calibration on an Android device is shown in Figure 1 .

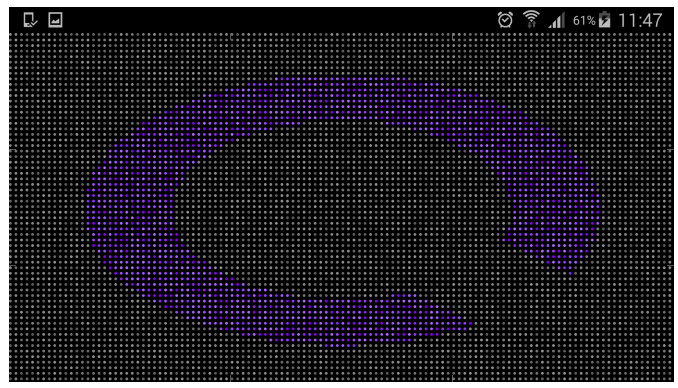

Figure 1: Screenshot of a single mobile calibration task on Android.

Once a person has completed the calibration, a personalized ICV simulation LUT is generated for them. The measurements from the calibration are also recorded in a unique user profile in case the LUT is accidentally deleted. The LUT and/or profile can be transferred to any other mobile running our simulation app to allow easy access to the personalized ICV simulations without requiring the person with ICV to recalibrate on each individual device.

\subsection{Mobile Implementation}

We developed an application for the Android 4.1 operating system on a Samsung Galaxy S5 development platform. We used OpenCV (opencv.org) to gain access to individual frames from the mobile's rear-facing camera, and RenderScript to parallelize access to the personalized ICV simulation LUT. During real-time simulation, write-access to the LUT is not needed; parallel read-only access allows RenderScript to look-up the personalized ICV simulation colour for multiple pixels simultaneously.

To help reduce LUT generation time and app memory footprint, we opted to use RGB666 (six bits each for red, green, and blue) instead of the standard RGB888 (eight bits each). No differences were visible when visually comparing RGB666 and RGB888 simulation results, so we opted for the lower-footprint version.

In addition to personalized ICV simulations, we also provided standard dichromatic simulations for protanopia, deuteranopia, and tritanopia, as well as monochromacy to allow someone to use the system immediately without having to obtain a personalized ICV colour vision profile. We did include personalized ICV simulation profiles directly in our evaluation deployment (see Section 5), so similar profiles could be included in any future commercial release.

\section{COMPARATIVE EVALUATION}

The personalized ICV simulation approach has previously been shown to be significantly more precise than standard dichromatic simulations 11 by comparing ICV participant colour vision test scores and non-ICV participant test scores when using ICV simulations. However this was shown for only the limited sets of colours used in colour vision tests.
To expand our understanding of the quality of our personalized simulation approach, we conducted a follow-on quantitative evaluation in which we measured the difference in simulation between our personalized approach and the state-of-the-art in adjustable ICV simulations (developed by Machado, Oliveira, and Fernandes [19]). Machado's adjustable simulation algorithm is described in an online tutoria ${ }^{3}$ that outlines their simulation approach and provides a comprehensive set of RGB-to-RGB multiplication matrices that permit a straightforward implementation of their simulation technique.

Choosing the matrix for a given type (protan, deutan, tri$\tan$ ) and severity ( 0.0 for no ICV, up to 1.0 for dichromacy, in increments of 0.1 ) of ICV, allows simulation of that type and severity. The individual R,G, and B values for a given pixel are defined as a vector that is multiplied with the selected matrix (and clamped if necessary) to create a new vector containing the simulated $R, G$, and $B$ values.

As the direct correlation between Machado's severity levels and the severity level measured by our calibration technique is unknown, we compared our simulation using a fixed severity of known type to all severity levels of the same type for Machado's simulation. Based on our previous experience with our calibration, we chose a mid-severity ICV value of 35.0 for each of protan and deutan ICVs, and compared each of these to all severities of Machado's adjustable simulation for the corresponding type of ICV. We calculated the Euclidean distance in CIE L* $\mathrm{u}^{*} \mathrm{v}^{*}$ colour space (an approximation of perceptual difference 32]) between our simulation result and Machado's simulation result for every RGB666 colour for each level of severity and selected the severity that resulted in the lowest average Euclidean distance.

Machado's severity level that resulted in the minimum average Euclidean distance between our simulation technique and theirs was at severity 0.3 for both protan and deutan. At this severity, the minimum average distance was 12.46 CIE $\mathrm{L}^{*} \mathrm{u}^{*} \mathrm{v}^{*}$ colour space units for protan and 13.12 CIE $\mathrm{L}^{*} \mathrm{u}^{*} \mathrm{v}^{*}$ units for deutan. These values suggest that there is substantial agreement between the two simulation techniques; assuming that a distance of 2.3 represents the JustNoticeable Difference (JND) point between any two colours (14] citing 25]), the average disagreement between the two simulation techniques is approximately 5-6 JNDs. To put this in context, mapping the sRGB colour space into the CIE L* $\mathrm{u}^{*} \mathrm{v}^{*}$ (using a D65 whitepoint) results in an $\mathrm{L}^{*}$ range of 0-100 (43 JNDs), a $\mathrm{u}^{*}$ range of -83 to 175 (112 JNDs), and $\mathrm{a} \mathrm{v}^{*}$ range of -134 to 107 (105 JNDs).

The distribution of distances for the protan and deutan personalized and adjustable simulations is shown in Figure2 For each distribution, over $90 \%$ of the distances are less than 21 CIE L* $\mathrm{u}^{*} \mathrm{v}^{*}$ units, but there is a long tail extending to 65 for protan and 63 for deutan.

So where do the differences lie between our personalized simulation technique and Machado's adjustable simulation technique? Upon further investigation, we discovered two situations that result in differences:

1. Large Shift-Potential Colours: The two techniques differ in the level of modification for colours that have the potential for substantial shifting during simulation. Our personalized technique tends to shift these colours towards their dichromatic counterpart less ag-

\footnotetext{
$\sqrt[3]{\text { www.inf.ufrgs.br/ oliveira/ }}$
} 


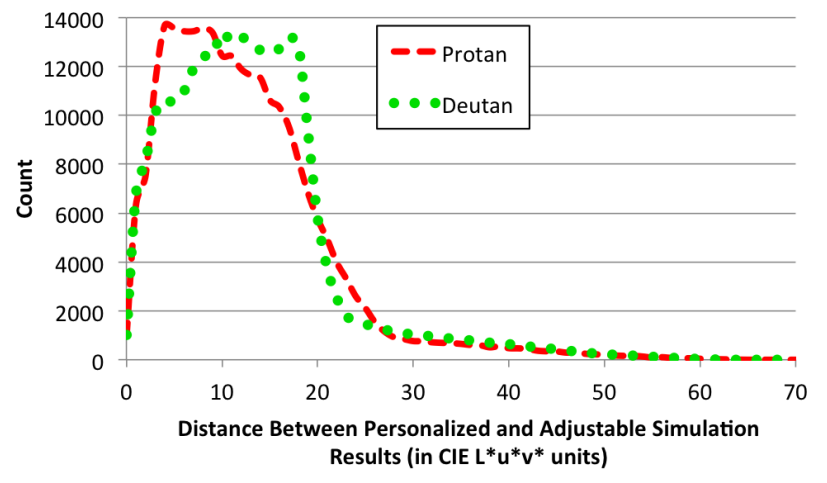

Figure 2: Histogram of differences between adjustable simulation and personalized simulation results for every RGB666 colour.

gressively than Machado's algorithm. This is illustrated on the right-hand side of Figure 3 , which shows Machado's (blue lines) more aggressive shifting of reds and purples compared to our personalized simulation technique (yellow lines).

2. Small Shift-Potential Colours: The two techniques also differ for colours that are close to the yellows, greys, and blues that colours are shifted toward during simulation. These colours appear to be shifted more aggressively by our personalized simulation technique compared to Machado's adjustable simulation. This is illustrated on the left-hand side of Figure 3, which shows Machado's (blue lines) less aggressive shifting of blues and greens compared to our personalized simulation technique (yellow lines).

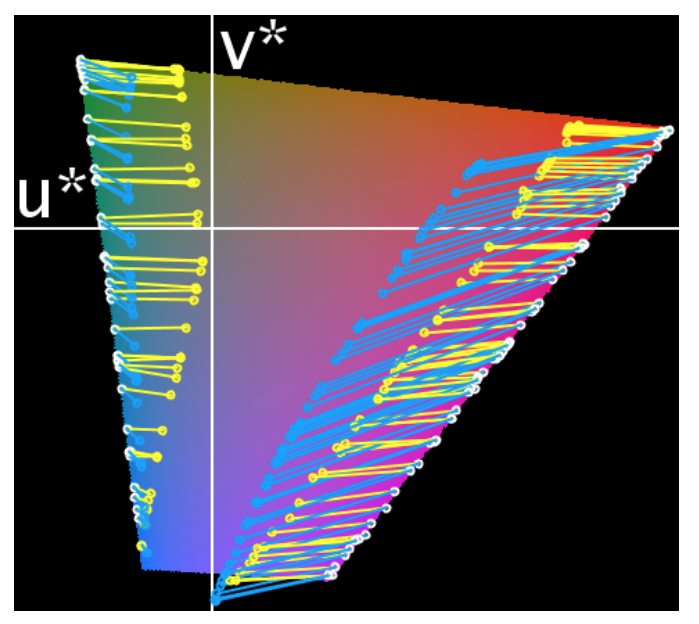

Figure 3: Illustration of shift amounts for our personalized simulation technique (protan, 35.0) and Machado's adjustable simulation technique (protan, 0.3). Original colours are white circles, personalized simulation results and shift lines in yellow, adjustable simulation results and shift lines in blue.

The difference between these two simulation techniques appears to be our reliance upon fixed shift distances in our simulation technique and the use of scalable shift distances in Machado's technique. In recent discussions about this difference with colour scientists at the Norwegian Colour and Visual Computing Laboratory ${ }^{4}$, we have identified strengths and weaknesses of each approach and concluded that more work is needed. In spite of these differences, the shift directions (towards blues, yellows, and greyscales) and shift magnitudes for each simulation technique largely agree, so we leave further examination and possible refinement of ICV simulation techniques as future work.

\section{USER EVALUATION}

In addition to the comparison evaluation described above, we also conducted a user evaluation in which we used our app to provide personalized simulations of three participants with ICV to nine participants with typical colour vision.

\subsection{Participants}

Twelve participants were recruited for the study. Three participants had some degree of ICV and nine participants had typical colour vision. The colour vision of all participants was assessed using the HRR Polychromatic Plates 13.

The participants with ICV were all male between the ages of 22 and 28, each with a different type of ICV: 1 mild protan, 1 mild deutan, and 1 near-monochromatic (degenerative incomplete achromatopsia) with self-reported perception of only extreme blues and yellows. The participants with typical colour vision consisted of two females (aged 22 and 25) and seven males (aged 18 to 29).

\subsection{Experimental Design}

This evaluation comprised an in-the-wild deployment of our app that allows people with typical colour vision to see the real-world colours as someone with ICV sees them.

To help capture what the participants with typical colour vision experienced during the deployment, we added a 'Capture Image' feature to our app. This feature allowed participants to record both the original and ICV-simulated versions of whatever was currently being displayed by our application. Each pair of images (original and simulated) were recorded to the device's local storage and retrieved later for analysis. We asked each participant to take pictures of anything they found of interest.

In conjunction with the image gathering step, we discussed the process with each typical-colour vision participant in an informal interview process. This interview was centered on discussing each participant's general impressions of experiencing a personalized simulation of ICV, as well as specific details about the circumstances and reasoning for each image they captured.

\subsection{Procedure}

Participants with ICV were recruited first. For each of these participants, we tested their colour vision using the HRR plates [13] and then had them run the calibration procedure twice on our development Samsung Galaxy S5 device. We generated simulations for each calibration for each participant and visually compared them (along with the ICV participant) to ensure the calibration accurately reflected the ICV participant's vision. The simulations appeared very similar for each ICV participant, so we arbitrarily chose one

${ }^{4}$ NTNU (Norwegian University of Science and Technology), Gjøvik, Norway 
of each pair to serve as the simulation profile for each ICV participant. This profile was then incorporated into our app to be deployed to participants with typical colour vision.

Participants with typical colour vision were recruited next. Each of these participants was tested using the HRR plates (to verify they had no ICV). Then we installed our simulation app on their personal Android device, and loaded one of the ICV profiles gathered from the participants with ICV. One ICV profile was chosen for each non-ICV participant to give them a concentrated experience of a single person's ICV vision as this reflects the likely use case in real world deployment (e.g., a mother trying to understand the colour vision of her son with ICV).

With nine participants with typical colour vision and three participants with ICV, a 3:1 assignment ratio would have been natural. However, near-monochromatic vision is both much rarer than the protan and deutan vision of the other two ICV participants, and it is also very similar to seeing in greyscale. As many people will have had the opportunity to see greyscale versions of photographs and television, we opted to assign the near-monochromatic simulation to a single typical colour vision participant, and four of these participants to each of the deutan and protan simulations.

Participants with typical colour vision then used our simulation app in their day-to-day lives for between seven and ten days. Participants were encouraged to capture photos of anything interesting they experienced during this time. After this deployment period, we met with each participant individually to collect their images, and to speak with each participant about their experiences using the simulation.

Although we originally wanted to hold the data collection and discussion session collectively with all twelve participants (ICV and typical colour vision), timing and scheduling constraints of our participants made this impossible. We still believe that there would be great value in coordinating a joint discussion session with ICV and non-ICV participants (as done in the previous work on personalized simulations [11]), so plan to do this in the future.

\subsection{Results}

We met with each typical colour vision participant individually to transfer their photos, discuss the context around each photo they took, and to gather their thoughts via an open-ended questionnaire.

\subsubsection{Photos Gathered}

Overall, our nine participants recorded 461 unique images (with original and simulated versions of each) over the span of 7-10 days. The maximum captured by one participant was 125 , the minimum was 16 (mean of 51).

After transferring the photos, we discussed each photo with the participants, using "What does this picture show?" and "Why did you take this picture?" as prompts to gather their reasoning behind each photo. Using this feedback, we classified the photos into categories, which represent many day-to-day challenges identified by previous work 27,11 : $44 \%$ of photos were of products, $21 \%$ of photos were of challenges around the home, $17 \%$ of photos were within the context of work or education, $10 \%$ were outside, and $7 \%$ of photos were of leisure activities (e.g., watching sports).

The most informative photos taken by participants were ICV simulations of shopping for food and products. Participants identified the very visual nature of selecting food (e.g., ensuring something is fresh or ripe), and most participants noticed that fresh fruit and vegetables looked discoloured and not very fresh, as illustrated in Figure 4 , bottom.

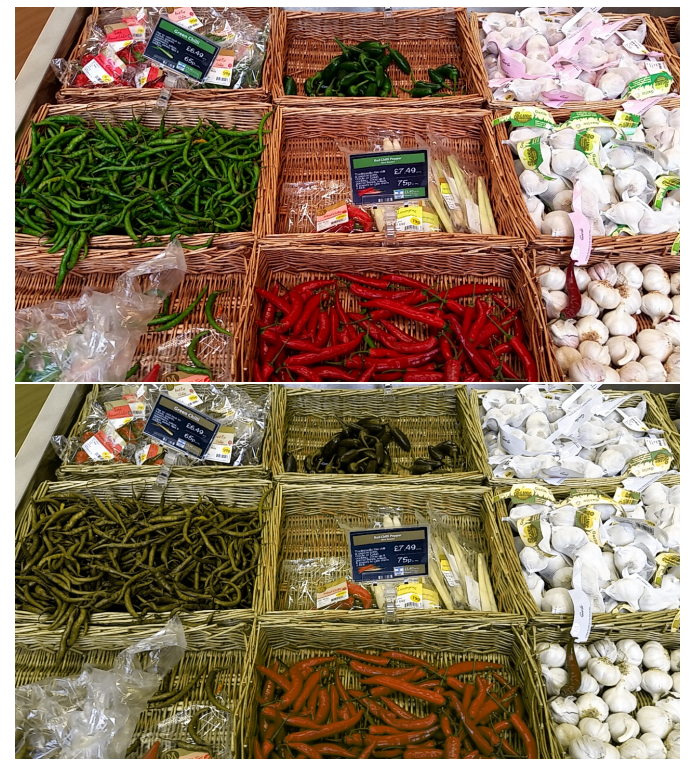

Figure 4: Original image (top), and deutan ICV simulated image (bottom) of a produce stand.

Another theme related to shopping was the reduced visual distinctiveness of product packaging. Product packaging colours are often chosen to be eye-catching or familiar to shoppers, but the yellow and green patterns on the dental dog biscuits shown in Figure 5 are much less apparent in the CVD simulation.

A final theme that emerged from the discussions with participants about their photos was how the use of colour to distinguish between products did not work well in their simulations. An example of this is shown in Figure 6, in which the lime (green) and lemon (yellow) versions of body wash are nearly indistinguishable in the CVD simulation image.

\subsubsection{Questionnaire Reponses}

As previously stated, the protan and deutan ICV simulations were given to four typical colour vision participants each, with the near-monochromatic ICV simulation given to a single typical colour vision participant. Because of the differences between the red-green and the monochromatic simulation, we treat feedback from these two sets of participants separately, first reporting the responses from those with the protan and deutan simulations. Our interview questionnaire contained four questions (discussed here) and one additional question (presented in Section 6). We list each question here and briefly summarize user responses.

What did you immediately notice about colours in the simulation you received? Participants with protan and deutan simulations all commented on the apparent 'dullness' or 'muted' nature of colours when viewed through the simulation. Participants with the protan simulation identified green as a particularly challenging colour as it typically appeared brown or yellow in the simulation, leading to confusion. Participants also perceived that the following sets of colours are often difficult to distinguish: reds, pinks, 


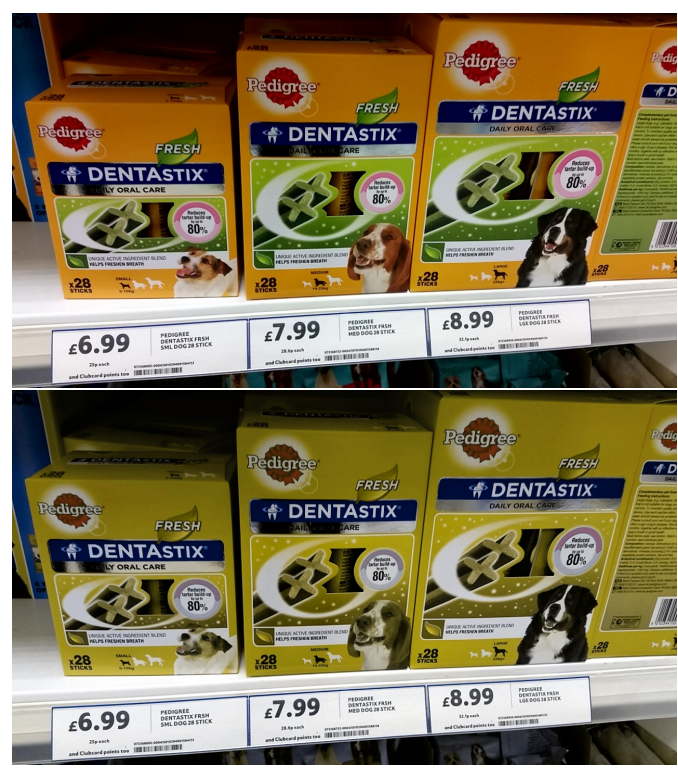

Figure 5: Original (top) and protan ICV simulation (bottom) of dog dental biscuits packaging. Packaging colours are not very distinctive in the simulation.

and greys; blues and purples; and greens and yellows, which agrees with the experience reported by people with ICV 27].

Did you find any specific areas of interest? As reflected in the photo results listed above, participants identified food (e.g., produce) and packaging (of food and products) as interesting because of the stark differences between the simulation and their own experience. Two participants with the protan simulation identified the appearance of their own simulated skin colour as a surprise. The greyness of Caucasian skin tones, as well as the invisibility of rashes and burns have also been previously identified 11]. The simulations helped one deutan simulation participant realize that their workplace could present challenges, and another identified choosing and applying make-up as a difficulty.

Did anything surprise you? Participants with protan simulations highlighted the difficulty in telling the ripeness of bananas as well as repeated how food generally appeared to not be fresh and was quite 'off-putting'. Those with deutan simulations did not identify anything specific, but reiterated how the over-reliance on colour makes many general tasks more difficult for people with ICV.

Did the application improve your understanding of ICV? Every participant readily agreed that our app was an effective means of building understanding. One participant remarked that "it was pretty amazing".

It is difficult to identify whether these findings are attributable to seeing personalized ICV simulations rather than standard dichromatic ICV simulations, and did not design our study to test this. However, we hold that a more precise simulation will naturally afford greater empathy and understanding in people with typical vision; knowing that they see colours as another human being sees them must offer greater personal value than using an ill-fitting standard simulation.

\subsubsection{Near-Monochromatic Results}

The typical vision participant who received the near-monochromatic ICV simulation had an experience that was at

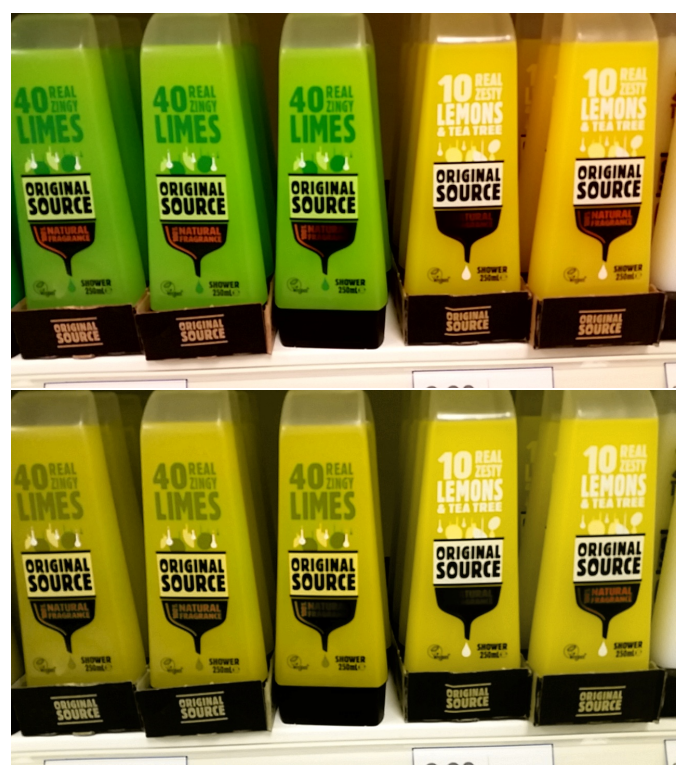

Figure 6: Original (top), and protan ICV simulation (bottom) of green (lime) and yellow (lemon) body wash. The two body wash types are not visually distinct in the simulation.

once both similar and different from that of the participants who had the protan and deutan simulations.

There was an immediate shock value when looking at this simulation, as it was almost entirely devoid of colour save for anything that was intensely yellow or blue (to the nonICV participant). This resulted in almost all of the photos gathered with this simulation profile being entirely greyscale - it was difficult for the typical colour vision participant to find situations in which any colour remained in the simulation (see Figure 7 for one example of successfully capturing some residual colour). The challenges (and shock) experienced by the participant using this simulation are evident in some of their responses to the questionnaire: "There weren't any [colours]. At all. It was like the world was a black and white movie. I noticed there was some yellow and blue but there really wasn't much of it unless it was very bright. Most of the time it was just a total absence of all colours."

In spite of the immediate shock and disbelief, this nonICV participant did come to understand much more about ICV and the challenges that such an extreme form of ICV would give rise to: "How could someone possibly have that as their vision? You would miss out on so many colours and so much detail on things. It must be horrible to have to deal with that all day every day!" and "It looks like everything is an issue with this type of ICV. It's pretty hard to tell things apart when they're just shades of grey."

"When I thought of colourblindness [ICV], I never realised that it could be as bad as that...it was really just like taking pictures in black and white mode."

\section{DISCUSSION}

Our findings suggest that providing a real-time mobile personalized simulation technique to people with typical colour vision can indeed help them gain insights into the challenges that ICV presents. This applies to both more-common forms of ICV (inherited protan and deutan ICV) as well as 


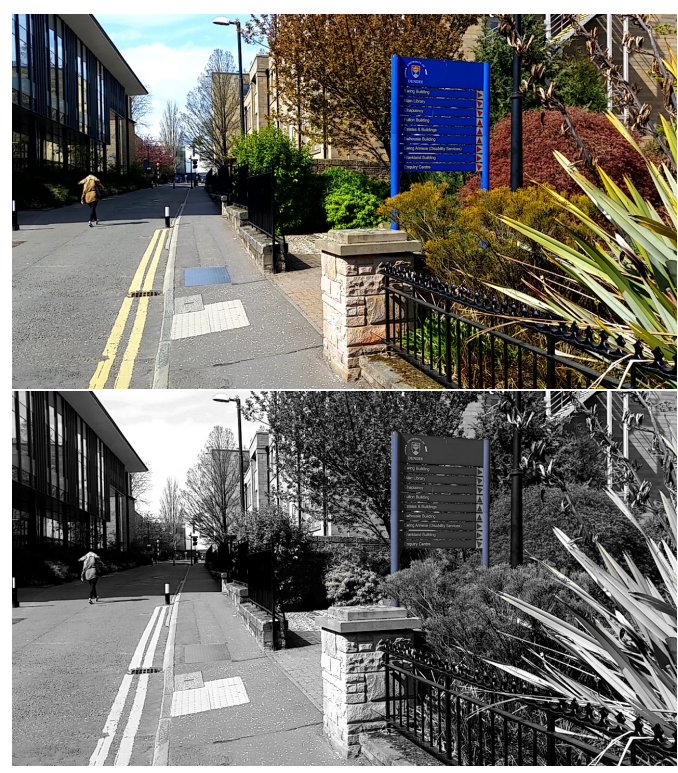

Figure 7: Original (top), and near-monochromatic simulation (bottom) of a blue sign and yellow street lines. Only the bright blue of the sign posts remain in the simulation.

less-common forms of ICV (as represented by our participant with degenerative incomplete achromatopsia).

The real-time nature of our app allowed participants to use ICV simulations in their day-to-day lives, and was less cumbersome than a simulation technique based on static photos (as used in previous studies). Similarly, the mobile nature of the app allowed participants to take the simulation with them anywhere, although some participants commented on the awkwardness of using a mobile app in this way in public - taking pictures of produce displays or road signs is not a 'normal' activity, and can easily lead to suspicion from others, and self-conscious feelings in the user.

That said, it is arguable that the 'personalized' feature of our tool was not adequately evaluated in our user study. We wanted to provide non-ICV participants with a rich and deep experience of a single individual's ICV, so participants did not get to experience a wide range of ICV types and severities. However, we did provide default dichromatic simulations to participants in addition to the personalized simulation, so participants may have experimented with the different simulations, although none explicitly commented on doing this. Our suspicion is that a personalized ICV simulation provided specific value to the non-ICV participants, in that they knew that their simulation was another human being's actual colour vision. Indeed, this was reflected in the near-monochromatic simulation participant's comments when they expressed disbelief that someone could actually have such severe colour vision loss.

Further to this, we also asked non-ICV participants if they identified anything that they anticipated would be a problem for people with ICV, but learned from using our app that it actually was not an issue. Participants with protan simulations identified that road signs were still quite vibrant (partly due to the 'mild' nature of their ICV simulations), and participants with deutan simulations identified traffic lights as not actually presenting a problem. Participants also commented that distinguishing between money and football (soccer) uniforms was less challenging than they anticipated.

\subsection{Extensions \& Future Work}

Using an in-situ real-time simulation in which people with typical colour vision were able to see the world through someone else's eyes proved to be a valuable and insightful mechanism for improving understanding of ICV. In the future, we would like to extend this work in four ways.

First, participants commented on the cumbersome nature of having to hold their mobile device to view the ICV simulations. To help address this, we are developing wearable ICV 'goggles' which provide a hands-free mechanism for 'seeing the world through someone else's eyes', similar to 1$]$ but using personalized ICV simulations.

Second, the original personalized simulation paper explored how ICV simulation helped non-ICV participants understand the ICV of someone close to them (friend or family) [11. In this paper, we explored enhancing the understanding of a general population that did not necessarily know the participants with ICV. We anticipate that there is great value in providing personalized ICV simulations to designers (to aid in the development of ICV-friendly designs) and teachers (who will have children with ICV in their classes), so are currently making plans to explore the value of ICV simulation with these specialist populations.

Third, our personalized ICV simulation technique and Machado's adjustable ICV simulation technique [19] both provide a mechanism for allowing people with typical colour vision to explore the range of severities of ICV. In recent demonstrations of these techniques to people with little previous understanding of ICV, we found that providing a simulation with a tuneable amount of severity (ranging from none to full dichromatism) was a valuable learning mechanism seeing how colours shift as ICV severity increases helped people understand ICV more clearly. We are currently investigating this through continued public demonstrations, and plan to explore this empirically in the future.

Finally, we are looking to provide our system to the public, either by releasing our product commercially or by providing our codebase for free download. In the meantime, we invite interested parties to contact us directly so we can provide access to our software.

\section{CONCLUSION}

In this paper, we describe extending personalized simulations of ICV to real-time operation on mobiles by incorporating automatic LUT generation, and using these LUTs to increase the speed of the personalized simulation algorithm. We also present a comparison of our simulation to an existing adjustable ICV simulation technique - showing that they are similar, but also that more work is needed. Finally, we present findings from an in-the-wild user study in which participants with typical colour vision demonstrated their increased understanding through the collection of photos illustrating problematic uses of colour for people with ICV.

To further contribute to our goal of increasing awareness of ICV, we are now working on extending our simulation further by building and testing our hands-free ICV 'goggles', exploring how tuneable ICV simulations can contribute to learning, and working with specialist populations to help increase their understanding of ICV. 


\section{REFERENCES}

[1] H. C. Ates, A. Fiannaca, and E. Folmer. Immersive simulation of visual impairments using a wearable see-through display. In Proceedings of the Ninth International Conference on Tangible, Embedded, and Embodied Interaction, pages 225-228. ACM, 2015.

[2] J. Birch. Diagnosis of defective colour vision. Elsevier Health Sciences, 2001.

[3] J. Birch. Worldwide prevalence of red-green color deficiency. Journal of the Optical Society of America A, 29(3):313-320, 2012.

[4] H. Brettel, F. Viénot, and J. D. Mollon. Computerized simulation of color appearance for dichromats. Journal of the Optical Society of America A, 14(10):2647-2655, October 1997.

[5] B. L. Cole. The handicap of abnormal colour vision. Clinical $\& 5$ Expirimental Optometry, 87(4-5):258-275, July 2004.

[6] I. R. Davies, G. Laws, G. G. Corbett, and D. J. Jerrett. Cross-cultural differences in colour vision: Acquired 'colour-blindness' in africa. Personality and Individual Differences, 25(6):1153-1162, 1998.

[7] F. Dick, S. Semple, R. Chen, and A. Seaton. Neurological deficits in solvent-exposed painters: a syndrome including impaired colour vision, cognitive defects, tremor and loss of vibration sensation. QJM: An International Journal of Medicine, 93(10):655-661, 2000.

[8] D. R. Flatla, A. R. Andrade, R. D. Teviotdale, D. L. Knowles, and C. Stewart. ColourID: Improving colour identification for people with impaired colour vision. In Proceedings of the 33rd Annual ACM Conference on Human Factors in Computing Systems, pages 3543-3552. ACM, 2015.

[9] D. R. Flatla and C. Gutwin. Improving calibration time and accuracy for situation-specific models of color differentiation. In ASSETS '11: Proceedings of the 13th International ACM SIGACCESS Conference on Computers and Accessibility, pages 195-202, 2011.

[10] D. R. Flatla and C. Gutwin. Situation specific models of color differentiation. TACCESS: ACM Transactions on Accessible Computing, 4(3):13:1-13:44, 2012.

[11] D. R. Flatla and C. Gutwin. "So that's what you see!" building understanding with personalized simulations of colour vision deficiency. In ASSETS '12: Proceedings of the 14th International ACM SIGACCESS Conference on Computers and Accessibility, pages 167-174, 2012.

[12] D. S. Fong, F. B. Barton, G. H. Bresnick, and E. T. D. R. S. R. Group. Impaired color vision associated with diabetic retinopathy: Early treatment diabetic retinopathy study report no. 15. American Journal of Ophthalmology, 128(5):612-617, 1999.

[13] L. H. Hardy, G. Rand, and M. C. Rittler. HRR polychromatic plates. Journal of the Optical Society of America, 44(7):509-521, 1954.

[14] J. Heer and M. Stone. Color naming models for color selection, image editing and palette design. In Proceedings of the SIGCHI Conference on Human Factors in Computing Systems, pages 1007-1016. ACM, 2012.

[15] C. Kaufman-Scarborough. Seeing through the eyes of the color-deficient shopper: Consumer issues for public policy. Journal of Consumer Policy, 23(4):461-492, December 2000.

[16] K. Knoblauch, F. Vital-Durand, and J. L. Barbur. Variation of chromatic sensitivity across the life span. Vision Research, 41(1):23-36, 2001.

[17] S. Kondo. A computer simulation of anomalous color vision. Color Vision Deficiencies, pages 145-159, 1990.

[18] O. Lagerlöf. Tricyclic psychopharmaca and colour vision. Documenta Ophthalmologica Proceedings Series, 1982.

[19] G. M. Machado, M. M. Oliveira, and L. A. F. Fernandes. A physiologically-based model for simulation of color vision deficiency. IEEE Transactions on Visualization and Computer Graphics, 15(6):1291-1298, 2009.

[20] G. W. Meyer and D. P. Greenberg. Color-defective vision and computer graphics displays. IEEE Computer Graphics and Applications, 8:28-40, September 1988.

[21] M. Pacheco-Cutillas, D. Edgar, and A. Sahraie. Acquired colour vision defects in glaucoma-their detection and clinical significance. British Journal of Ophthalmology, 83(12):1396-1402, 1999.

[22] K. Reinecke, D. R. Flatla, and C. Brooks. Enabling designers to foresee which colors users cannot see. In Proceedings of the 34th Annual ACM Conference on Human Factors in Computing Systems, pages 2693-2704. ACM, 2016.

[23] O. Sacks. An anthropologist on Mars: Seven paradoxical tales. Vintage, 2012.

[24] M. E. Schneck and G. Haegerstrom-Portnoy. Color vision defect type and spatial vision in the optic neuritis treatment trial. Investigative ophthalmology 8 visual science, 38(11):2278-2289, 1997.

[25] G. Sharma and R. Bala. Digital color imaging handbook. CRC press, 2002.

[26] J. A. B. Spalding. Confessions of a colour blind physician. Clinical and Experimental Optometry, 87(4-5):344-349, 2004.

[27] J. M. Steward and B. L. Cole. What do color vision defectives day about everyday tasks? Optometry and Vision Science, 66(5):288-295, May 1989.

[28] M. Stone. A field guide to digital color. CRC Press, 2013.

[29] G. W. Tigwell, D. R. Flatla, and N. D. Archibald. ACE: A usable tool for designing accessible colour palettes. TACCESS: ACM Transactions on Accessible Computing, 0(0):30 pages, In submission 2016.

[30] F. Viénot, H. Brettel, L. Ott, A. B. M'Barek, and J. D. Mollon. What do colour-blind people see? Nature, 376:127-128, July 131995.

[31] B. A. Wandell. Foundations of vision. Sinauer Associates, 1995.

[32] G. Wyszecki and W. S. Stiles. Color Science: Concepts and Methods, Quantitative Data and Formulae. Wiley New York, 2nd edition, 2000.

[33] C. D. Zippe, A. W. Kedia, K. Kedia, D. R. Nelson, and A. Agarwal. Treatment of erectile dysfunction after radical prostatectomy with sildenafil citrate (viagra). Urology, 52(6):963-966, 1998. 